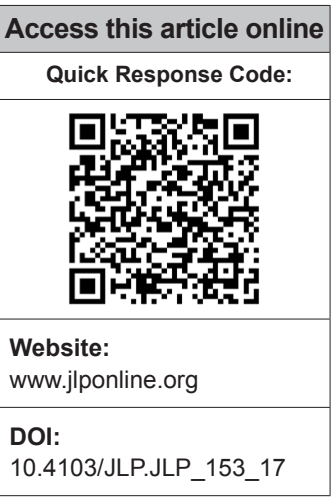

Department of Pathology,

Dr. Ram Manohar Lohia Hospital, PGIMER, New Delhi, India

Address for correspondence: Dr. Manjari Kishore, Department of Pathology, Dr. Ram Manohar Lohia Hospital, PGIMER, Room No. $310,3^{\text {rd }}$ Floor, OPD Building, Baba Kharak Singh Maarg, New Delhi - 110001 , India. E-mail: drmanjarik@gmail.

Submission: 09-10-2017 Accepted: 02-01-2018

\title{
Unusual presentation of renal cell carcinoma: A rare case report
}

\author{
Manjari Kishore, Devender Singh Chauhan, Shruti Dogra
}

\section{Abstract:}

Cutaneous and intraoral metastasis from any malignancy is not common. Cutaneous spread is usually noted in $5 \%-10 \%$ of high-grade malignancies, as in carcinoma breast, lung, colon, ovary, and malignant melanoma. Only $4.6 \%$ cases of cutaneous spread are from renal cell carcinoma (RCC). Intraoral spread from RCC is much rarer with an incidence of approximately $1 \%$ of all malignant oral tumors, noted sometimes in tongue, palate, buccal mucosa, gingiva, and lips. RCC is a highly aggressive tumor which requires early diagnosis for increasing the chance of cure. In our case, a 54-year-old male presented with swelling over upper lip, scalp, and retromolar area, which on histopathology and immunohistochemistry revealed clear cell carcinoma compatible with metastatic RCC.

Key words:

Cutaneous, hypernephroma, immunohistochemistry, intraoral, scalp

\section{Introduction}

$\mathrm{R}_{\mathrm{a}}$ enal cell carcinoma (RCC), also known as hypernephroma, is the most common type of renal tumor noted in adults, especially in the age group of $50-70$ years. ${ }^{[1,2]}$ The usual metastatic foci from RCC are noted in lungs, bone, liver, adrenal glands, and brain. Metastatic deposits of RCC to retromolar area and cutaneous regions are rarely noted. Oral cavity metastasis from RCC portends a very poor prognosis representing a widespread disease. The rich vascular proliferation noted in RCC is the reason behind increased hematogenous spread leading to distant metastasis. We present an unusual case of multiple distant cutaneous and retromolar metastasis from RCC in a 54-year-old male.

\section{Case Report}

A 54-year-old male presented to the hospital with swelling over upper lip, scalp, and retromolar region [Figure 1a and b]. He gave a history of significant weight loss over the past

This is an open access article distributed under the terms of the Creative Commons Attribution-NonCommercial-ShareAlike 3.0 License, which allows others to remix, tweak, and build upon the work non-commercially, as long as the author is credited and the new creations are licensed under the identical terms.

For reprints contact: reprints@medknow.com
3 months. The exophytic lesion over upper lip measured $2 \mathrm{~cm} \times 1.8 \mathrm{~cm}$ [Figure 2a]. The surface of swelling was lobulated with focal hemorrhagic crusting with visible pulsation. On palpation, the swelling was firm, nontender, nonpulsatile, and did not bleed on touch. Two small swellings measuring $1.8 \mathrm{~cm} \times 1.4 \mathrm{~cm}$ and $1.2 \mathrm{~cm} \times 1 \mathrm{~cm}$ each were noted on the scalp, which grossly mimicked some vascular lesion. A large, firm, diffuse growth measuring $6 \mathrm{~cm} \times 4.5 \mathrm{~cm} \times 3.2 \mathrm{~cm}$ was also noted in the right lower retromolar area involving the gums with rich vascularity and bled on touch. Oral hygiene of the patient was poor.

On proper evaluation, the patient told that he was diagnosed with widespread RCC with multiple skeletal metastasis 2 months back. Complete blood count and biochemical investigations of the patient were within normal limit. The clinical differential of these lesions included metastasis from renal carcinoma, angiosarcoma, pyogenic granuloma, Kaposi sarcoma, and amelanotic melanoma.

An 18 F-fluorodeoxyglucose (FDG) positron emission tomography/computed

How to cite this article: Kishore M, Chauhan DS, Dogra S. Unusual presentation of renal cell carcinoma: A rare case report. J Lab Physicians 2018;10:241-4. 
tomography (PET/CT) whole body was done. PET-contrast-enhanced CT revealed heterogeneously enhancing exophytic nodular mass lesion with large central necrotic component, arising in the lower pole of right kidney. Multiple FDG avid metastatic mediastinal and bilateral hilar lymphadenopathy was noted along with subpleural and parenchymal bilateral pulmonary nodules. A slightly irregular heterogeneously enhancing mass was also noted in the left adrenal. Multiple skeletal metastasis was also noted. Surgery was not done in view of widespread metastasis, and he was kept on palliative therapy. Noncontrast $\mathrm{CT}$ head did not reveal any evidence of metastasis.

Histopathologic examination from the different swellings as described above showed similar morphology. Sections revealed mildly hyperkeratotic stratified squamous epithelium with focal ulceration and overlying fibrinosuppurative exudate [Figure 2b]. Dermis showed a malignant tumor composed of malignant epithelial cells arranged in solid nests, occasional glandular pattern, and few clusters [Figure 2c]. Cells showed small round hyperchromatic nuclei, prominent nucleoli, and moderate amount of clear to eosinophilic cytoplasm in a background of rich vascular network [Figure 2d]. Immunohistochemistry revealed positivity for CD10 and Vimentin [Figure 2e and f] and negativity for HMB-45, S100, smooth muscle actin and Desmin. Based on the clinical, radiological, histomorphological, and immunohistochemical findings, a final diagnosis of multiple cutaneous and intraoral metastatic RCC was made.

In view of extensive metastasis, surgical intervention was not done. The patient was given chemotherapy with oral sorafenib and radiotherapy for the cutaneous lesions. The patient was alive and stable till the last follow-up.

\section{Discussion}

RCC comprises approximately $2 \%-3 \%$ of adult malignancies. ${ }^{[1-3]}$ Usually patients present with vague flank pain, sometimes hematuria, and palpable abdominal mass. The common organs where metastasis from RCC occurs are lungs, regional lymph nodes, bone, liver, adrenal glands, contralateral kidney, and brain. Cutaneous presentation of RCC is not commonly noted. Cutaneous metastasis is seen in $2.8 \%-4.4 \%$ of skin malignant carcinoma, of which metastasis from RCC accounts for only $6 \% .^{[2-4]}$ The usual site of metastasis in head and neck region are scalp followed by abdominal region. Cases have been reported in regions such as tongue, palate, gingiva, nasal cavity, maxillary sinus, larynx, parotid, and thyroid glands. ${ }^{[1-3]}$ Cases of metastasis to upper lip and intraoral region, i.e., retromolar area have rarely been reported. ${ }^{[2,3]}$
RCC metastasis to skin can be noted before the diagnosis of the primary tumor, but usually, it is found after few months or years of nephrectomy or after months of diagnosis of RCC. In our case, the patient was diagnosed with RCC with widespread metastasis to skeletal system just 2 months back. In view of metastasis at the time of presentation, surgery was not advised and he was started with chemotherapeutic drugs. He again presented to the hospital with lesion on upper lip, followed by the development of multiple lesions on scalp and right retromolar area. It has been found in literature that $20 \%-$ $30 \%$ of patients who have had nephrectomy for RCC will develop distant metastasis. ${ }^{[3-5]}$ This aggressive nature of this neoplasm is the reason for poor survival in these patients.

The pathogenesis of cutaneous metastasis from visceral malignancies involves the direct invasion of skin tissue overlying the malignant renal mass. ${ }^{[6]}$ The other routes involved in dissemination are arterial, venous, and lymphatics. A possibility of spread through Batson's plexus and thoracic duct is considered in metastasis to head and neck areas. ${ }^{[2,3]}$

Cutaneous presentation can be varied and lead to wide differential diagnoses. Usually, cutaneous metastases appear as solitary, red to purple deposits. The increased vascularity leads to suspicion of vascular lesions such as pyogenic granuloma, hemangioma, and Kaposi's sarcoma. Radiology is of little significance in diagnosing skin metastasis, however, other location of metastasis can be recognized. ${ }^{[3,5]}$

Fine needle aspiration as a diagnostic modality in these oral and cutaneous lesions involves elevated risk of bleeding and is noted in majority of patients. Hence, biopsy is the gold standard for diagnosing metastasis and should be done in all situations with adequate precaution. Histopathology with light microscopy alone cannot help much in differentiating RCC metastasis from other clear cell tumors and is challenging, especially from clear cell malignancies of salivary glands which also shows nests of clear cells divided by thin fibrous septae..$^{[2-5]}$

Other differentials are - clear cell variant of odontogenic tumors (Pindborg tumor), primary or metastatic melanoma, and other metastatic clear cell carcinomas. Here, immunohistochemistry comes into play. RCC exhibits focal cytokeratin positivity whereas it is diffuse in salivary gland carcinoma. RCC also shows exhibit strong expression of Vimentin which helps in making the diagnosis, especially in case with the previous history of renal malignancy.

Treatment in oral and cutaneous metastasis from RCC is usually palliative and involves a multidisciplinary 
approach. Excision of skin lesion is recommended if face or scalp is involved. In advanced, inoperable cases, chemotherapy is given. However, the prognosis of patients with oral and cutaneous involvement is poor. Although symptomatic relief can be given in few cases. Azam et al. ${ }^{[7]}$ reported a case where surgical debridement was done for growing metastatic tongue lesion which caused difficulty in swallowing to the patient. Following surgery, radiotherapy was given to oral cavity to treat remaining foci. Kyan and Kato ${ }^{[8]}$ also reported surgical resection in a lingual mass followed by interferon- $\alpha$ and interleukin-II therapy. Recently, newer agents, such as vascular endothelial growth factor inhibitors - bevacizumab, sunitinib, sorafenib, have been widely used in patients with widespread RCC metastasis. ${ }^{[2,-9]}$ Trials have shown positive effect with prolongation of survival in the patients.

A thorough clinical examination of patients with RCC is must due to the possibility of the appearance of

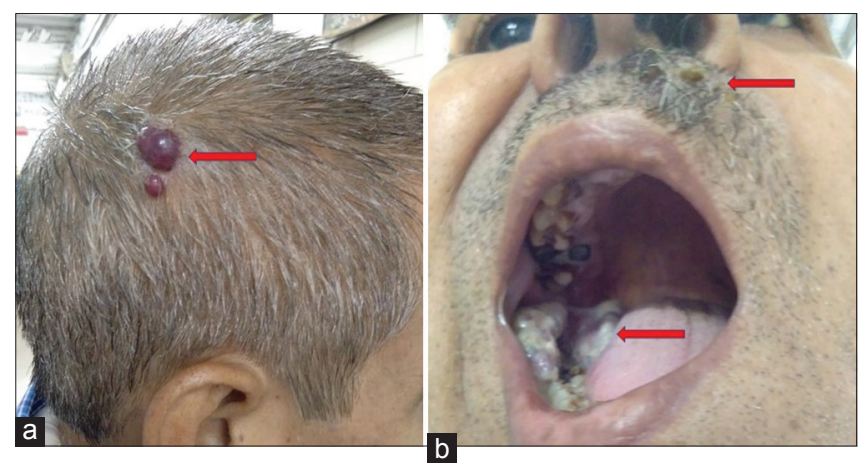

Figure 1: (a and b) Photographs showing multiple swellings over scalp, upper lip, and retromolar region (marked by red arrow) metastasis at multiple sites. A differential of metastatic deposit of RCC must be kept in setting of new-onset nodules, ulcers, tumors with vascular appearances, especially in a patient with the previous history of RCC or nephrectomy.

\section{Conclusion}

Cutaneous and intraoral metastasis from RCC can sometimes pose a challenge in cases where there is no history of previous renal pathology, and at times histomorphology can be deceptive mimicking other clear cell carcinomas from salivary glands. Immunohistochemistry plays a very important role in helping the pathologists arriving at a correct diagnosis. While evaluating the cutaneous and intraoral lesions in an adult patient, metastatic lesions should always be kept in mind and primary tumor should be carefully looked for. Hence, a proper clinicopathological correlation is of utmost importance in making a correct diagnosis of this metastatic malignancy.

\section{Declaration of patient consent}

The authors certify that they have obtained all appropriate patient consent forms. In the form the patient(s) has/ have given his/her/their consent for his/her/their images and other clinical information to be reported in the journal. The patients understand that their names and initials will not be published and due efforts will be made to conceal their identity, but anonymity cannot be guaranteed.

\section{Financial support and sponsorship} Nil.

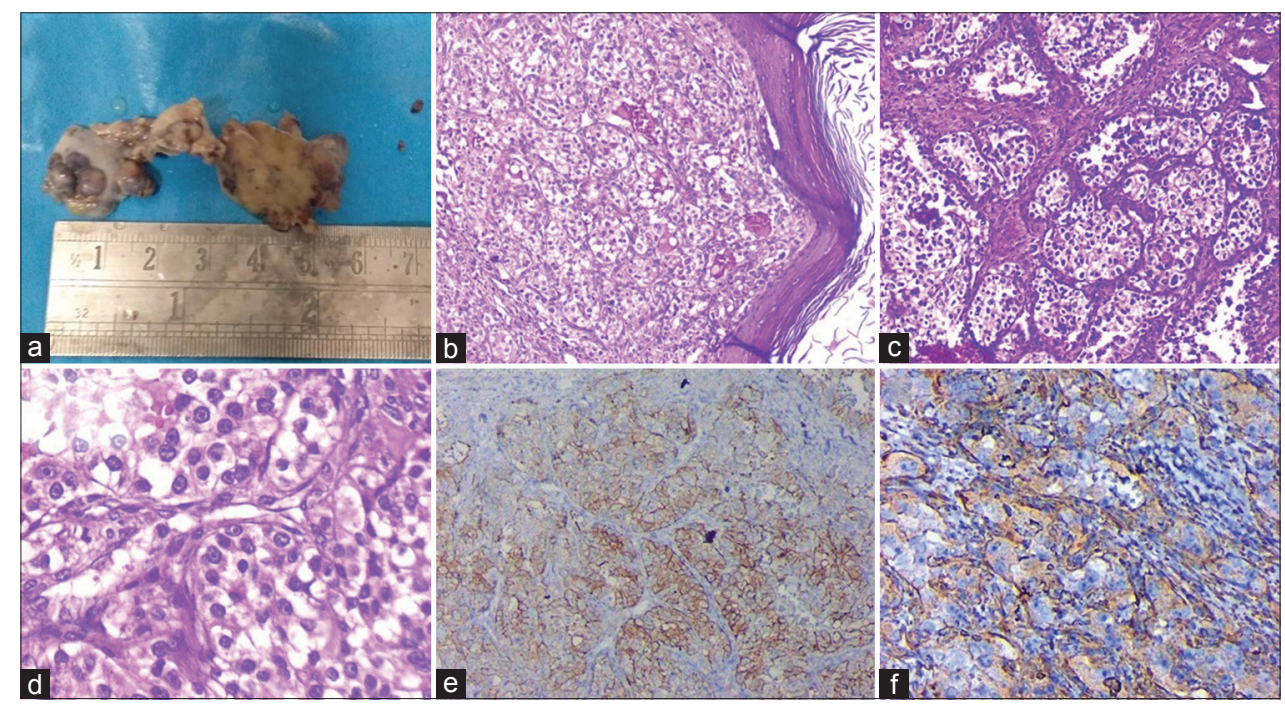

Figure 2: (a) Gross photograph of exophytic lesion over upper lip measuring $2 \mathrm{~cm} \times 1.8 \mathrm{~cm}$. (b) Photomicrograph showing mildly hyperkeratotic epidermis with subepithelium showing nest and lobules of malignant epithelial cells separated by fibroconnective tissue (H and E - x100). (c and d) Malignant epithelial cells showing small round hyperchromatic nuclei, prominent nucleoli and moderate amount of clear to eosinophilic cytoplasm in a background of rich vascular network ( $\mathrm{C}-\mathrm{H}$ and $\mathrm{E}, \times 200 ; \mathrm{d}-\mathrm{H}$ and $\mathrm{E}, \times 400)$. (e and f) Cells showing immunoreactivity for CD10 (e) and Vimentin (f) 


\section{Conflicts of interest}

There are no conflicts of interest.

\section{References}

1. Flanigan RC, Campbell SC, Clark JI, Picken MM. Metastatic renal cell carcinoma. Curr Treat Options Oncol 2003;4:385-90.

2. Kotak A, Merrick G. Presentation of metastatic renal cell carcinoma as a lip lesion. J Surg Case Rep 2014;2014:pii: rju083.

3. Arrabal-Polo MA, Arias-Santiago SA, Aneiros-Fernandez J, Burkhardt-Perez P, Arrabal-Martin M, Naranjo-Sintes R, et al. Cutaneous metastases in renal cell carcinoma: A case report. Cases J 2009;2:7948.

4. Pritchyk KM, Schiff BA, Newkirk KA, Krowiak E, Deeb ZE. Metastatic renal cell carcinoma to the head and neck. Laryngoscope
2002;112:1598-602.

5. Porter NA, Anderson HL, Al-Dujaily S. Renal cell carcinoma presenting as a solitary cutaneous facial metastasis: Case report and review of the literature. Int Semin Surg Oncol 2006;3:27.

6. Koga S, Tsuda S, Nishikido M, Matsuya F, Saito Y, Kanetake H, et al. Renal cell carcinoma metastatic to the skin. Anticancer Res 2000;20:1939-40.

7. Azam F, Abubakerr M, Gollins S. Tongue metastasis as an initial presentation of renal cell carcinoma: A case report and literature review. J Med Case Rep 2008;2:249.

8. Kyan A, Kato SN. Renal cell carcinoma metastatic to the base of tongue: A case report. Hinyokika Kiyo 2004;50:791-3.

9. Fukuda M, Miyata M, Okabe K, Sakashita H. A case series of 9 tumors metastatic to the oral and maxillofacial region. J Oral Maxillofac Surg 2002;60:942-4. 